\title{
Risco para doenças cardiovasculares e fatores associados em idosos não institucionalizados com doença de Alzheimer
}

\author{
Risk for cardiovascular diseases and associated factors in non-institutionalized elderly people with
} Alzheimer's disease

DOI: $10.37111 /$ braspenj.2020354007

Carolinne Teixeira da Silva

Aline Jacoski de Oliveira Kruger da Silva ${ }^{2}$

Caroline do Vale de Campos

Carla Zanelatto ${ }^{3}$

Juliana Sartori Bonini ${ }^{4}$

Caryna Eurich Mazur ${ }^{5}$

\section{Unitermos:}

Antropometria. Estado nutricional. Demência. Doenças cardiovasculares.

\section{Keywords:}

Anthropometry. Nutritional status. Dementia. Cardiovascular diseases.

\section{Endereço para correspondência:}

Caryna Eurich Mazur

Universidade Estadual do Centro-Oeste Ringgold standard institution - Nutrição

Rua Simeão Varela de Sá, 3 - Vila Carli - Guarapuava,

PR, Brasil - CEP 85040-080

Email: carynanutricionista@gmail.com

\section{Submissão}

5 de maio de 2020

Aceito para publicação

9 de dezembro de 2020

\begin{abstract}
RESUMO
Introdução: As doenças crônicas degenerativas são comuns em idosos, dentre elas destacam-se doença cardiovascular (DCV) e doença de Alzheimer (DA). Ambas afetam o estado nutricional com mudanças na ingestão e no hábito alimentar. Dessa maneira, o objetivo desse estudo foi avaliar o risco para DCV e outros fatores em idosos com DA. Método: Estudo retrospectivo realizado entre 2015 a 2017, em Guarapuava - PR, com aplicação da mini avaliação nutricional (MAN), cálculo e classificação do índice de massa corporal (IMC). Para avaliação de risco para DCV, foi aferida a circunferência da cintura (CC), razão cintura estatura (RCE) e índice de conicidade (IC). Foi aplicado o recordatório de 24 horas (R24). Foram empregados testes de associação e regressão logística ordinal, com valores significantes quando $p<0,05$. Resultados: Participaram do estudo 39 pacientes, com média de idade de 78,9 anos, 53,8\% eram do sexo masculino, $41 \%$ encontravam-se em eutrofia pelo IMC e 53,8\%, bem nutridos pela MAN. O risco para DCV foi considerado alto em $36,8 \%$ dos pacientes, segundo a CC. Houve diferença significativa entre os idosos com risco e alto risco, quando comparadas à pontuação da MAN, IMC, RCE e IC $(p<0,05)$. A dieta foi considerada adequada para carboidrato, proteína e lipídeo em 48,7\%, 61,5\% e $48,7 \%$, respectivamente. O consumo de proteína aumentou o risco para DCV em 2,57 vezes $(I C=1,26-5,24 ; p<0,05)$. Conclusões: $O$ risco foi alto para DCV tanto pela CC quanto pela RCE e IC. Os pacientes estavam em sua maioria com dieta hipocalórica e os valores de proteína se relacionaram com o risco para DCV.
\end{abstract}

\section{ABSTRACT}

Introduction: Degenerative chronic diseases are common in the elderly, among which stand out cardiovascular disease (CVD) and Alzheimer's disease (AD). Both affect nutritional status with changes in intake and eating habits. Thus, the aim of this study was to evaluate the risk for CVD and other factors in elderly with AD. Methods: Retrospective study conducted between 2015 and 2017, in Guarapuava - PR, Brazil, where it was applied the mini nutritional assessment (MAN), calculated and classified the body mass index (BMI). To assess CVD risk, waist circumference (WC), waist height ratio $(\mathrm{WH}+\mathrm{R})$ and conicity index $(\mathrm{Cl})$ were measured. The 24 -hour recall was applied (R24). Association tests and ordinal logistic regression were used with significant values when $p$ $<0.05$. Results: 39 patients participated in the study, the average age was 78.9 years, $53.8 \%$ were male, $41 \%$ were eutrophic by BMI and $53.8 \%$ well-nourished by MAN. The risk for CVD was considered high at $36.8 \%$ according to WC. There was a significant difference between at-risk and high-risk elderly compared to MAN, BMI, WHtR and $\mathrm{Cl}$ scores $(\mathrm{p}<0.05)$. The diet was considered adequate for carbohydrate, protein and lipid in $48.7 \%, 61.5 \%$ and $48.7 \%$, respectively. Protein intake increased the risk for CVD by 2.57-fold $(\mathrm{Cl}=1.26-5.24 ; \mathrm{p}<0.05)$. Conclusions: The risk was high for CVD by both WC, WH+R and $\mathrm{Cl}$. Most patients were on a low-calorie diet and protein values were related to CVD risk.

1. Nutricionista, Universidade Estadual do Centro-Oeste (UNICENTRO). Guarapuava, PR, Brasil.

2. Nutricionista, Prefeitura Municipal de Turvo, Turvo, PR, Brasil.

3. Nutricionista, doutora em Saúde Coletiva, Universidade Federal Fronteira Sul (UFFS), Departamento de Nutrição, Realeza, PR, Brasil.

4. Farmacêutica, doutora em Bioquímica, Universidade Estadual do Centro-Oeste (UNICENTRO), Departamento de Farmácia, Guarapuava, PR, Brasil.

5. Nutricionista, doutora em Medicina Interna, Universidade Estadual do Centro-Oeste (UNICENTRO), Departamento de Nutrição, Guarapuava, PR, Brasil. 


\section{INTRODUÇÃO}

O envelhecimento populacional é um fenômeno global. No Brasil, os dados apontados pelo Instituto Brasileiro de Geografia e Estatística indicam para o futuro um "país de idosos" devido à transição demográfica, com aumento significativo da população que envelhece e, também, da expectativa de vida' ${ }^{1}$. Na diferenciação por sexo, as mulheres já ultrapassaram 78,8 anos de idade como expectativa de vida e os homens chegaram aos 71,6 anos de idade?.

As doenças crônico-degenerativas destacam-se, com predominância em indivíduos idosos, entre elas está a doença cardiovascular (DCV). De acordo com a Organização Mundial da Saúde (OMS), cerca de 17,5 milhões de pessoas morrem todos os anos vítimas de DCV, sendo a principal causa de morte no mundo. Além da idade, outros fatores de risco estão relacionados, como hipertensão, diabetes mellitus, fumo, dislipidemias, sedentarismo e obesidade 2 .

Atualmente, 30,4\% dos óbitos no Brasil são por DCV. É evidente a associação entre a mortalidade por DCV e o avançar da idade, uma vez que a grande maioria das mortes por essa categoria de doenças ocorre em pessoas acima de 65 anos. Fatores dietéticos têm papel importante na gênese das DCV associada à aterosclerose, doença coronariana, acidente vascular cerebral e insuficiência cardíaca ${ }^{1,3}$.

Ainda, no envelhecimento, a nutrição fica condicionada às mudanças fisiológicas, metabólicas e na capacidade funcional. Além disso, a população idosa é heterogênea pela diversidade social, cultural, econômica e idade fisiológica, que resultam na alteração das necessidades nutricionais e no desenvolvimento de outras doenças crônicas não transmissíveis, como diabetes mellitus, DCV e obesidade, importantes fatores de risco também no desenvolvimento da doença de Alzheimer (DA) ${ }^{3}$.

A DA é uma enfermidade neurodegenerativa irreversível, atualmente é a causa principal de demência, que atinge comumente os idosos ${ }^{4}$. Pode ser dividida em 3 fases, leve, moderada e grave, de acordo com a sua progressão. Na fase leve, os sintomas são perda de memória, desorientação no tempo e no espaço, perda da espontaneidade e da iniciativa, alterações de humor e de concentração. A fase moderada apresenta dificuldades na linguagem, em aprender coisas novas e lidar com situações inesperadas, agitação, ansiedade, não reconhecimento de pessoas e lugares. Na última fase da doença, o paciente pode perder totalmente a sua independência, ficando acamado, necessitando de cuidados permanentes, na medida em que surgem problemas ao nível da deglutição, sinais neurológicos, incontinência fecal e urinária 5 .

Pacientes com DA necessitam de um aumento do aporte calórico devido a algumas alterações de demanda energética e agitação. Com o avanço da doença, ocorre uma mudança do hábito alimentar, aumentando a ingestão de carboidratos simples e a diminuição de proteínas, devido à dificuldade na mastigação, deglutição e diminuição da digestibilidade gástrica, que podem acometer estes pacientes ${ }^{6}$.
As condições nutricionais de idosos portadores de DA são afetadas de maneira irreversível, por uma provável inaptidão na aceitação, mastigação, deglutição e assimilação dos alimentos. Para tal, uma alimentação adequada ao longo do prognóstico da doença é essencial na preservação do estado clínico, físico e nutricional, elevando ao máximo as funções orgânicas vitais, além de poder contribuir para a estabilidade e melhorar a qualidade de vida do paciente ${ }^{7}$.

Portanto, devido às alterações fisiológicas consequentes no processo de envelhecimento, os idosos ficam mais suscetíveis ao desenvolvimento de $D C V$, que ocorrem principalmente em indivíduos cujos hábitos alimentares são, em sua maioria, constituídos por alimentos ricos em gorduras e carboidratos, de alto valor calórico, pobre em micronutrientes e baixa qualidade nutricional. Os idosos diagnosticados com DA têm maior dificuldade em se alimentar de forma equilibrada, devido às falhas de memória e demência progressivas que a doença causa. Diante disso e tendo em vista a escassez de estudos que avaliaram o risco para DCV e fatores associados em idosos com DA, se faz necessária a avaliação antropométrica, dietética e clínica, visando ao monitoramento e à melhora da qualidade de vida dessa população em particular. Assim, o objetivo deste trabalho foi avaliar o risco para DCV, fatores clínicos, dietéticos e antropométricos em idosos com DA em Guarapuava, Paraná.

\section{MÉTODO}

Trata-se de um estudo retrospectivo, realizado com banco de dados da equipe multidisciplinar que atende a idosos com DA, avaliados durante os anos de 2015 a 2017 . A coleta de dados foi realizada com idosos residentes na cidade de Guarapuava, Paraná, Brasil, com diagnóstico clínico de DA realizado por médico neurologista ou geriatra. Os pacientes foram convidados a participar da pesquisa, por intermédio da Associação de Estudos, Pesquisa e Auxílio às pessoas com Alzheimer (AEPAPA), com sede no mesmo município (http:// www.aepapa.org.br.

A mini avaliação nutricional (MAN) foi aplicada para avaliar o risco nutricional dos pacientes com DA. A MAN é um instrumento composto por medidas antropométricas, avaliação global e de alimentação. A pontuação de 24 a 30 pontos indicou estado nutricional normal, 17 a 23,5 pontos risco de desnutrição e menos de 17 pontos indicou desnutrição ${ }^{8}$.

$\bigcirc$ peso foi aferido com balança digital com capacidade de $150 \mathrm{~kg}$, estando o paciente com o mínimo de roupa possível e com os pés descalços. Na impossibilidade de aferir o peso, o mesmo foi estimado conforme Chumlea et al. ?. A altura do paciente foi aferida com fita inelástica fixada em parede sem rodapés, ou na impossibilidade foi aferida altura recumbente na cama em que o paciente estava. Foi calculado o índice de massa corporal (IMC) com o peso e altura do paciente e classificado conforme Lipschitz ${ }^{10}$, sendo considerado magro $\left(<22 \mathrm{~kg} / \mathrm{m}^{2}\right)$; eutrófico $\left(22-27 \mathrm{~kg} / \mathrm{m}^{2}\right)$ e com excesso de peso $\left(>27 \mathrm{~kg} / \mathrm{m}^{2}\right)$. 
Para estimar o risco para $\mathrm{DCV}$, foi aferida a circunferência da cintura (CC), no ponto médio aproximado entre a margem inferior da última costela palpável e a parte superior da crista ilíaca, ao final de uma expiração normal. A CC foi classificada conforme recomendações estabelecidas pela OMS ${ }^{11}$. Foi questionada aos cuidadores dos idosos a presença de comorbidades associadas, como diabetes mellitus e hipertensão arterial sistêmica.

A razão cintura estatura (RCE) foi realizada a partir da CC (em centímetros) dividida pela medida da estatura (centímetros). A classificação foi feita seguindo critérios de Ashwell e Gibson'2, onde o valor maior de 0,5 é sugerido como ponto de corte para o risco de desenvolvimento de DCV, em indivíduos de ambos os sexos.

Para o cálculo do índice de conicidade (IC) utilizou-se a fórmula preconizada por Valdez ${ }^{13}$, a classificação de risco para DCV foi determinada conforme valores acima de 1,25. Determinou-se o IC por meio das medidas de peso, estatura e CC, utilizando-se uma equação matemática.

A taxa metabólica basal (TMB) e gasto energético total (GET) foram calculados de acordo com o preconizado por Harris e Benedict ${ }^{14}$. Para o cálculo do GET, utilizou-se fator de injúria 1,4; fator térmico 1,0; e fator atividade para paciente confinado ao leito: 1,2; acamado, porém móvel: 1,25; e para paciente que deambula: 1,3.

Para análise dietética, foi aplicado ao cuidador do paciente o recordatório de 24 horas, um método retrospectivo em que o cuidador relatou tudo que o paciente consumiu no dia anterior. Avaliaram-se as calorias totais e percentuais de carboidrato, proteína e lipídeo, com auxílio do software DietWin $2008^{\circledR}$. De acordo com as Dietary Reference Intakes (DRIs), o percentual recomendado para ingestão de proteínas diárias é de $10 \%$ a $35 \%$, carboidratos de $45 \%$ a $65 \%$ e lipídeos de $20 \%$ a $35 \%$ do GET ${ }^{15}$.

O Clinical Dementia Rating (CDR) foi utilizado para analisar o grau de demência dos idosos, onde avalia 0 comportamento, além da influência das perdas cognitivas na capacidade de realizar adequadamente as atividades de vida diária. O CDR é constituído por três categorias numéricas, onde: 1 - indica demência leve, 2 - demência moderada e 3 corresponde a demência grave ${ }^{16}$.

As variáveis foram analisadas descritivamente e por análise inferencial. Inicialmente, para verificar a normalidade da amostra foi aplicado o teste de Shapiro Wilk. Para associações não-paramétricas foi utilizado o teste de qui-quadrado e teste de Kruskal-Wallis. Além disso, foi calculada a análise da variância (ANOVA) para dados paramétricos e, em resultados significativos, foi aplicado o post-hoc de Bonferroni para testar o dado divergente. Ainda, as associações foram testadas em análises bivariadas. Um modelo de regressão logística ordinal foi utilizado para estimar os valores das razões de chance $(R C)$ e respectivos intervalos de confiança (IC95\%) para cada um dos níveis do desfecho, simultaneamente, com programa SPSS ${ }^{\circledR} 22.0$.

Nesta pesquisa foram respeitados todos os princípios éticos estabelecidos na Resolução n ${ }^{\circ}$ 466/2012, a pesquisa foi previamente aprovada pelo Comitê de Ética em Pesquisa da Universidade Estadual do Centro Oeste (UNICENTRO), sob o número 2.200.768/2017.

\section{RESULTADOS}

Foram inclusos no estudo 39 pacientes idosos com diagnóstico clínico de DA. A média de idade observada foi $78,97 \pm 7,17$ anos, a maioria $(53,8 \%)$ era do sexo masculino, e nesse mesmo percentual foram classificados na faixa etária de 65-79 anos. De acordo com o IMC, $41 \%$ da amostra encontravam-se em eutrofia, conforme a MAN $53,8 \%$, foram classificados como bem nutridos. O risco para DCV foi alto, conforme a CC, para $36,8 \%$ dos pacientes. Já para as classificações de risco conforme RCE e IC, ambos perfizeram $84,2 \%$. Conforme apresentado na Tabela 1, não houve diferença estatística quando estratificados os grupos por faixa etária $(p>0,05)$.

Tabela 1 - Comparação de classificações de estado nutricional e risco para doenças cardiovasculares com a faixa etária de doente com Alzheimer. Guarapuava, 2018.

\begin{tabular}{|c|c|c|c|c|}
\hline Variáveis & Total & $\begin{array}{c}\text { 65-79 anos } \\
(\mathrm{n}=21)\end{array}$ & $\begin{array}{c}\geq 80 \text { anos } \\
(n=18)\end{array}$ & $p$-valor \\
\hline \multicolumn{5}{|l|}{ Classificação IMC } \\
\hline Magreza & $10(25,6)$ & $6(28,6)$ & $4(22,2)$ & 0,88 \\
\hline Eutrofia & $16(41,0)$ & $8(38,1)$ & $8(44,4)$ & \\
\hline Excesso de peso & $13(33,3)$ & $7(33,3)$ & $6(33,3)$ & \\
\hline \multicolumn{5}{|l|}{ Classificação MAN } \\
\hline Desnutrição & $5(12,8)$ & $3(14,3)$ & $2(11,1)$ & 0,79 \\
\hline $\begin{array}{l}\text { Risco de } \\
\text { desnutrição }\end{array}$ & $12(33,3)$ & $6(28,6)$ & $7(38,9)$ & \\
\hline Bem nutrido & $21(53,8)$ & $12(57,1)$ & $9(50,0)$ & \\
\hline \multicolumn{5}{|l|}{ Classificação CC ${ }^{\star \star}$} \\
\hline Sem risco & $11(28,9)$ & $5(25,0)$ & $6(33,3)$ & 0,71 \\
\hline Risco & $13(34,2)$ & $8(40,0)$ & $5(27,8)$ & \\
\hline Alto risco & $14(36,8)$ & $7(35,0)$ & $7(38,9)$ & \\
\hline \multicolumn{5}{|c|}{ Classificação RCE } \\
\hline Sem risco & $6(15,8)$ & $3(15,0)$ & $3(16,7)$ & 0,64 \\
\hline Risco & $32(84,2)$ & $17(85,0)$ & $15(83,3)$ & \\
\hline \multicolumn{5}{|l|}{ Classificação IC ${ }^{\star \star}$} \\
\hline Sem risco & $6(15,8)$ & $2(10,0)$ & $4(22,2)$ & 0,38 \\
\hline Risco & $32(84,2)$ & $18(90,0)$ & $14(77,8)$ & \\
\hline
\end{tabular}

$\mathrm{CC}$ = circunferência da cintura; $\mathrm{IC}$ = índice de conicidade; $\mathrm{IMC}=$ índice de massa corporal; MAN = mini avaliação nutricional; RCE = razão cintura-estatura.

${ }^{a} Q u i-q u a d r a d o .{ }^{* *}$ ão foi aferida circunferência da cintura em um paciente.

Para as classificações de MAN, CC, RCE e IC foram considerados 38 indivíduos. 
De acordo com o estadiamento da DA, 35,9\% dos pacientes apresentaram CDR 1, que corresponde a DA leve, 33,3\% apresentaram CDR 2 ou DA moderada e $28,2 \%$ foram classificados em CDR 3 ou DA grave. Eram hipertensos $41 \%$ da amostra e 10,3\% eram diabéticos. Quando houve estratificação da amostra conforme o risco para DCV segundo a CC, pode-se perceber uma diferença significativa entre os idosos com risco e alto risco quando comparadas a pontuação da MAN, o IMC, a RCE e o IC $(p<0,05)$. Em contrapartida, os pacientes sem risco para DCV apresentaram consumo de proteína menor quando comparados aos grupos de risco $(p<0,05)$ (Tabela 2).

A média da TMB foi $749,04 \pm 214,13 \mathrm{kcal}$, já o GET foi $1326,59 \pm 385,28$ kcal. A maioria (59\%) dos pacientes apresentou adequação calórica em relação às necessidades energéticas no R24h. A dieta foi considerada adequada para carboidrato, proteína e lipídeo em 48,7\%, $61,5 \%$ e $48,7 \%$ da amostra, respectivamente. As variáveis idade, sexo, presença de hipertensão arterial sistêmica, diabetes mellitus, CDR, calorias totais, carboidrato e lipídeo não tiveram associação quando estratificadas conforme o risco para DCV de acordo com a CC. Entretanto, o consumo de proteína obteve uma associação 2,57 vezes maior quando o paciente apresentava risco para DCV $(I C=1,26-5,24 ; p<0,05)$. Quando comparados valores descritivos de proteína em gramas foi observado que o grupo com risco para DCV (média: 71, 12 27,83 gramas) foi significativamente maior que o grupo sem risco (média: 45,42 $\pm 13,59)(p<0,05)$ (Tabela 3).

Tabela 2 - Comparação dos valores médios das variáveis do estudo com o risco para doenças cardiovasculares em pacientes com doença de Alzheimer. Guarapuava, 2018.

\begin{tabular}{|c|c|c|c|c|c|}
\hline & \multicolumn{5}{|c|}{ Risco para DCV } \\
\hline & Total $(n=39)$ & Alto risco $(n=14)$ & Risco (n=13) & Sem risco $(n=11)$ & $p$-valor \\
\hline (média; DP) & $78,97 \pm 7,17$ & $79,29 \pm 7,82$ & $78,69 \pm 5,04$ & $79,64 \pm 8,84$ & $0,95^{\mathrm{a}}$ \\
\hline Feminino & $18(46,2)$ & $9(64,3)$ & $6(46,2)$ & $2(18,2)$ & $0,07^{b}$ \\
\hline Masculino & $21(53,8)$ & $5(35,7)$ & $7(53,8$ & $9(81,8$ & \\
\hline \multicolumn{6}{|l|}{$\mathrm{CDR}^{\star}(\mathrm{n} ; \%)$} \\
\hline Leve & $14(35,9)$ & $5(35,7)$ & $6(46,2)$ & $3(27,3)$ & $0,34^{\mathrm{b}}$ \\
\hline Pontuação MAN (média; DP) & $22,95 \pm 4,36$ & $24,08 \pm 3,14$ & $24,81 \pm 3,47^{£}$ & $20,64 \pm 3,43^{\varepsilon}$ & $0,01^{\mathrm{a}}$ \\
\hline Peso (média; DP) & $66,86 \pm 14,39$ & $72,10 \pm 17,59$ & $68,23 \pm 13,40$ & $59,42 \pm 7,63$ & $0,08^{\mathrm{a}}$ \\
\hline IMC (média; DP) & $25,31 \pm 4,33$ & $27,97 \pm 4,22^{\varepsilon}$ & $25,85 \pm 3,04$ & $21,75 \pm 3,17^{\varepsilon}$ & $0,001^{a}$ \\
\hline RCE (média; DP) & $0,58 \pm 0,07$ & $0,65 \pm 0,04^{\varepsilon}$ & $0,57 \pm 0,04$ & $0,49 \pm 0,04^{\varepsilon}$ & $0,001^{\mathrm{a}}$ \\
\hline IC (média; DP) & $1,34 \pm 0,11$ & $1,43 \pm 0,11^{£}$ & $1,32 \pm 0,07$ & $1,26 \pm 0,08^{\varepsilon}$ & $0,001^{a}$ \\
\hline Calorias totais (média; DP) & $1416,82 \pm 507,45$ & $1369,14 \pm 382,33$ & $1427,31 \pm 549,43$ & $1492,27 \pm 638,54$ & $0,84^{\mathrm{a}}$ \\
\hline
\end{tabular}

CDR = Clinical Dementia Rating; CHO = carboidrato; DCV = doenças cardiovasculares; DP = desvio-padrão; IC= índice de conicidade; IMC= índice de massa corporal; LIP= lipídio; MAN = Mini avaliação nutricional; PTN = proteína; RCE = razão cintura-estatura.

*Ausência de dados de CDR de um paciente.

${ }^{a}$ Análise da Variância (ANOVA); 'beste Kruskal-Wallis; EDiferem estatisticamente pelo teste de Bonferroni com 95\% de confiança. 
Tabela 3 - Resultado da regressão logística ordinal bivariada do risco para doenças cardiovasculares segundo idade, sexo, presença de comorbidades, escore da doença e variáveis dietéticas em doentes com Alzheimer. Guarapuava, 2018.

\begin{tabular}{|c|c|c|c|}
\hline & \multicolumn{2}{|c|}{ Risco para DCV } & \multirow[t]{2}{*}{ p-valor } \\
\hline & $\begin{array}{c}\operatorname{Sim}(n=27) \\
\mathrm{RC}-\mathrm{IC} 95 \%\end{array}$ & $\begin{array}{c}\text { Não }(n=11) \\
\text { RC - IC95\% }\end{array}$ & \\
\hline \multicolumn{4}{|l|}{ Idade } \\
\hline$\geq 80$ anos & 1 & 1 & 0,71 \\
\hline $65-79$ anos & $1,12(0,74-1,70)$ & $0,75(0,27-2,04)$ & \\
\hline \multicolumn{4}{|l|}{ Sexo } \\
\hline Feminino & 1 & 1 & 0,03 \\
\hline Masculino & $1,54(1,03-2,35)$ & $0,27(0,07-1,10)$ & \\
\hline \multicolumn{4}{|l|}{ Hipertensão } \\
\hline Sim & 1 & 1 & 0,72 \\
\hline Não & $1,10(0,74-1,64)$ & $0,79(0,28-2,24)$ & \\
\hline
\end{tabular}

\section{Diabetes}

\begin{tabular}{lccc} 
Sim & 1 & 1 & 0,67 \\
Não & $1,06(0,58-1,95)$ & $0,85(0,14-5,02)$ & \\
\hline CDR & & & \\
Leve + Moderada & $1,55(0,81-2,98)$ & $0,44(0,17-1,14)$ & 0,12 \\
Grave & 1 & 1 & \\
\hline
\end{tabular}

\section{Calorias totais}

\begin{tabular}{lccc} 
Adequadas & $0,78(0,52-1,16)$ & $1,94(0,61-6,19)$ & 0,29 \\
Não adequadas & 1 & 1 \\
\hline
\end{tabular}

\begin{tabular}{lccc}
\hline CHO & & & \\
Adequado & $1,08(0,72-1,62)$ & $0,83(0,31-2,27)$ & 0,72 \\
Não adequado & 1 & 1 & \\
\hline
\end{tabular}

\begin{tabular}{lccc}
\hline PTN & & & \\
Adequado & $2,57(1,26-5,24)$ & $0,13(0,03-0,52)$ & 0,001 \\
Não adequado & 1 & 1 & \\
\hline LIP & & & \\
Adequado & $1,25(0,83-1,89)$ & $0,57(0,20-1,63)$ & 0,48 \\
Não adequado & 1 & 1 & \\
\hline
\end{tabular}

$\mathrm{CDR}=$ Clinical Dementia Rating; $\mathrm{CHO}$ = carboidrato; IC= intervalo de confiança; $\mathrm{LIP}=$ lipídio; PTN = proteína; $\mathrm{RC}$ = razão de chances.

* Ausência de dados de risco para doença cardiovascular de um paciente.

\section{DISCUSSÃO}

Neste estudo, a maioria dos pacientes foi classificada com eutrofia e bem nutrida. O risco para DCV esteve presente em $36,8 \%$ dos pacientes, de acordo com o parâmetro tradicional (CC). A maioria estava em CDR 1 e boa parte da amostra era hipertensa.

A maior parte $(53,8 \%)$ dos pacientes deste estudo era do sexo masculino. Diferentemente do que apresenta a
Organização Mundial da Saúde e a Alzheimer's Disease International ${ }^{17}$, a prevalência de mulheres idosas que desenvolvem DA é maior que dos homens. Além disso, a maior taxa de mortalidade também está entre as mulheres, principalmente aquelas acima de 80 anos. Isso ocorre porque os homens apresentam maiores taxas de comorbidades associadas, assim como mortalidade precoce por DCV, enquanto as mulheres têm uma expectativa de vida maior, apresentando maior declínio funcional nas fases mais avançadas da vida ${ }^{18}$. Assim, esses dados não corroboram com esta pesquisa, iá que a maioria dos idosos avaliados era composta de homens.

No presente estudo, a maioria dos idosos avaliados apresentou eutrofia pelo IMC e foi classificada como bem nutrida pela MAN. Estudos comprovam o aumento do risco de desnutrição em idosos com DA, comparados àqueles que não são afetados por esta demência, devido à ocorrêcia de sinais e sintomas, principalmente da disfagia ${ }^{19}$. Entretanto, sabe-se que o IMC tem limitações em relação à composição corporal, não sendo capaz de avaliar o percentual de gordura corporal e massa muscular que os indivíduos apresentam ${ }^{4}$.

Neste estudo, a maioria dos idosos com DA apresenta risco para $D C V$, segundo os diferentes métodos utilizados. Acredita-se que esse resultado esteja associado a diferentes fatores de risco, entre eles a hereditariedade, sedentarismo, hábitos alimentares inadequados, baixa procura pelo serviço de saúde, tabagismo e a presença de comorbidades, como hipertensão e diabetes.

Considera-se a doença arterial coronariana como um fator de risco para DA, sendo ela caracterizada pela obstrução do fluxo sanguíneo do coração devido ao desencadeamento de aterosclerose das artérias subjacentes, causada pela hipertensão arterial sistêmica, tabagismo e diabetes mellitus ${ }^{20}$. De acordo com Lee et al. ${ }^{21}$, a prevalência de hipertensão arterial vem crescendo devido ao aumento da população geriátrica, e atinge cerca de um bilhão de pessoas no mundo, sendo o principal fator de risco para infarto agudo do miocárdio, acidente vascular cerebral, doença renal e insuficiência cardíaca. Assim como no presente estudo, identificou-se que grande parte dos pacientes avaliados era hipertensa, o que já pode ser um risco isolado para DCV.

Segundo Teixeira et al. ${ }^{18}$, a prevalência de DCV e diabetes é alta em idosos com DA. $O$ diabetes tem a capacidade de aumentar o risco para DCV em cinco vezes, por consequência de alguns fatores associados, como sedentarismo, dieta, obesidade, dislipidemias e hipertensão ${ }^{21}$.

Verificou-se que os idosos com DA apresentaram uma dieta hipocalórica em comparação às suas necessidades energéticas diárias. Contudo, há evidências científicas sobre a 
adesão da dieta mediterrânea que seria uma opção favorável para esses pacientes, pois relaciona-se com a diminuição do declínio cognitivo e da mortalidade, em pacientes portadores da DA. Ela consiste no aumento do consumo de ômega 3 proveniente de peixes, óleos vegetais, alimentos com baixo índice glicêmico, carboidratos complexos, carnes magras e baixa ingestão de açúcares, que atuam como efeito protetor da progressão da demência ${ }^{22}$.

No estudo realizado por Taylor et al. ${ }^{23}$, que avaliou a associação entre o consumo de alimentos com alto índice glicêmico e a carga de proteína beta-amieloide cerebral, em 128 idosos cognitivamente normais, evidenciou que uma dieta com alto índice glicêmico contribui para o acúmulo de amieloide cerebral e, como consequência, o desenvolvimento da DA. Entretanto, neste estudo, não foi possível avaliar o índice glicêmico da dieta.

Neste estudo, verificou-se que o consumo de proteína foi mais elevado nos pacientes que apresentaram risco para DCV. A proteína é um macronutriente que disponibiliza 4 $\mathrm{kcal} / \mathrm{grama}$ normalmente, os alimentos ricos em proteínas de alto valor biológico, principalmente os de origem animal, são também fontes de gordura saturada e colesterol e, se consumidos em excesso, são responsáveis pelo aumento do risco para $\mathrm{DCV}^{4}$.

Ainda, o desempenho cognitivo de idosos pode ser reduzido com o excesso de gordura saturada na dieta. Entretanto, o consumo de gorduras polinsaturadas, e a baixa ingestão de gorduras trans amenizam o declínio cognitivo ${ }^{4}$. A qualidade da dieta dos idosos não foi avaliada neste estudo, sendo um fator limitante para análise do consumo de lipídeos consumidos, de acordo com o recordatório de 24 horas.

Os dados relacionados às calorias totais, quantidades de proteínas, carboidratos e lipídeos desse estudo foram embasados no recordatório de 24 horas, um fator limitante para a adequada avaliação dietética dos idosos, exigindo memória do cuidador entrevistado. Além disso, sabe-se que há variações nos grupos de alimentos consumidos no dia-dia, sendo mais eficaz para avaliação dietética o diário de consumo alimentar, que não foi aplicado no presente estudo. Portanto, são necessários mais estudos relacionados à qualidade da dieta desses idosos portadores da DA para relacionar com os achados do presente estudo.

\section{CONCLUSÃO}

Foi observado que os pacientes com DA estavam em sua maioria com risco para DCV, conforme a CC, RCE e IC. Ainda, a maioria foi classificada em CDR 1. A dieta foi considerada hipocalórica, porém com valores médios de macronutrientes adequados. Grande parte da amostra estava em eutrofia pelo IMC e bem nutrida pela MAN. Dentre as associações apresentadas, destacam-se os valores adequados de proteína que tiveram relação com o risco para DCV. Os resultados aqui apresentados devem ser melhor elucidados com novas pesquisas longitudinais, multicêntricas e com maior número de pacientes, para confirmação das inferências aqui expostas.

\section{AGRADECIMENTOS}

Agradecemos à Associação de Estudos, Pesquisa e Auxílio às Pessoas com Alzheimer (AEPAPA), pelo espaço concedido para a realização desta pesquisa.

\section{REFERÊNCIAS}

1. Instituto Brasileiro de Geografia e Estatística. Estudos e pesquisas: informação demográfica e socioeconômica síntese de indicadores sociais. Uma análise das condições de vida da população brasileira. Rio de Janeiro: IBGE; 2015. [acesso em 1 de maio de 2020]. Disponível em: https://biblioteca.ibge.gov. br/visualizacao/livros/liv95011.pdf

2. Organização Mundial da Saúde (OMS). Relatório mundial de envelhecimento e saúde. WHO/FWC/ALC/15.01. Genebra: Organização Mundial da Saúde; 2015. [acesso em 1 de maio de 2020]. Disponível em: https://apps.who.int/iris/bitstream/ handle/10665/186468/WHO_FWC_ALC_15.01_por. pdf? sequence $=6$

3. Ivanski F, Nascimento LP, Fermino BL, Bonini JS, Silva WCFND, Valério JMS, et al. Nutritional evaluation of geriatric patients with Alzheimer's disease in Southern Brazil: case-control study. Nutr Hosp. 2018;35(3):564-9.

4. Doorduijn AS, van de Rest O, van der Flier WM, Visser M, de van der Schueren MAE. Energy and protein intake of Alzheimer's disease patients compared to cognitively normal controls: systematic. J Am Med Dir Assoc. 2019;20(1):14-21.

5. Solfrizzi V, Frisardi V, Seripa D, Logroscino G, Imbimbo BP, D'Onofrio G, et al. Mediterranean diet in predementia and dementia syndromes. Curr Alzheimer Res. 2011;8(5):520-42.

6. Ogawa Y, Kaneko Y, Sato T, Shimizu S, Kanetaka H, Hanyu H. Sarcopenia and muscle functions at various stages of Alzheimer disease. Front Neurol. 2018;9:710.

7. Tavares TE, Carvalho CMRG. Características de mastigação e deglutição na doença de Alzheimer. Rev CEFAC. 2012;14(1):122-37.

8. Vellas B, Villars H, Abellan G, Soto ME, Rolland Y, Guigoz Y, et al. Overview of the MNA: its history and challenges. J Nutr Health Aging. 2006;10(6):456-63.

9. Chumlea WC, Roche AF, Steinbaugh ML. Estimating stature from knee height for persons 60 to 90 years of age. J Am Geriatr Soc. 1985;33(2):116-20.

10. Lipschitz DA. Screening for nutritional status in the elderly. Prim Care. 1994;21(1):55-67.

11. World Health Organization. Obesity: preventing and managing the global epidemic. WHO Technical Report Series 894. Geneva: World Health Organization; 1998. [acesso em 1 de maio de 2020]. Disponível em: https://www.who.int/nutrition/ publications/obesity/WHO_TRS_894/en/

12. Ashwell M, Gibson S. Waist to height ratio is a simple and effective obesity screening tool for cardiovascular risk factors: analysis of data from the British National Diet and Nutrition Survey of adults aged 19-64 years. Obes Facts. 2009;2(2):97-103.

13. Valdez RA. A simple model-based index of abdominal adiposity. J Clin Epidemiol. 1991;44(9):955-6.

14. Harris JA, Benedict FG. Biometric studies of basal metabolism in man. Washington: Carnegie Institute of Washington; 1919. 
15. Trumbo P, Schlicker S, Yates AA, Poos M; Food and Nutrition Board of the Institute of Medicine, The National Academies. Dietary reference intakes for energy, carbohydrate, fiber, fat, fatty acids, cholesterol, protein, and amino acids. J Am Diet Assoc. 2002;102(11):1621-30.

16. MontañoMBMM, Ramos LR. Validade daversão em português da Clinical Dementia Rating. Rev Saúde Pública. 2005;39(6):912-7.

17. Alzheimer's Disease International. Relatório sobre a doença de Alzheimer no mundo. Resumo Executivo, 2009.

18. Teixeira JB, Souza Junior PR, Higa J, Theme Filha MM. Mortality from Alzheimer's disease in Brazil, 2000-2009. Cad Saude Publica. 2015;31(4):850-60.

19. González MB, Recio GM. Abordaje de la disfagia en enfermos de alzhéimer. Nutr Hosp. 2016;33(3):286.

20. Grace C, Clarke R, Goel A, Farall M, Watkins H, Hopewell JC. Lack of genetic support for shared aetiology of coronary artery disease and late-onset Alzheimer's disease. Sci Rep. 2018;8(1):7102.

21. Lee ES, Vedanthan R, Jeemon P. Quality improvement in cardiovascular disease care. In: Nugent R, ed. Source cardiovascular, respiratory, and related disorders. 3rd ed. Washington: The International Bank for Reconstruction and Development / The World Bank; 2017 Nov. Chapter 18.

22. Solfrizzi V, Custodero C, Lozupone M, Imbimbo BP, Valiani $\mathrm{V}$, Agosti P, et al. Relationships of dietary patterns, foods, and micro- and macronutrients with Alzheimer's disease and latelife cognitive disorders: a systematic review. J Alzheimers Dis. 2017;59(3):815-49.

23. Taylor MK, Sullivan DK, Swerdlow RH, Vidoni ED, Morris JK, Mahnken JD, et al. A high-glycemic diet is associated with cerebral amyloid burden in cognitively normal older adults. Am J Clin Nutr. 2017;106(6):1463-70.

Local de realização do estudo: Universidade Estadual do Centro-Oeste (UNICENTRO), Departamento de Nutrição, Guarapuava, PR, Brasil.

Conflito de interesse: Os autores declaram não haver. 